\title{
The Relationship among Tyrosine Decarboxylase and Agmatine Deiminase Pathways in Enterococcus faecalis
}

\begin{abstract}
Marta Perez ${ }^{1}$, Victor Ladero ${ }^{1}$ Beatriz del Rio', Begoña Redruello', Anne de Jong ${ }^{2}$, Oscar Kuipers², Jan Kok², M. Cruz Martin ${ }^{1}$, Maria Fernandez ${ }^{1 *}$ and Miguel A. Alvarez

${ }^{1}$ Dairy Research Institute, Consejo Superior de Investigaciones Cientificas (IPLA-CSIC), Asturias, Spain, ${ }^{2}$ Department of Molecular Genetics, Groningen Biomolecular Sciences and Biotechnology Institute, University of Groningen, Groningen, Netherlands
\end{abstract}

Enterococci are considered mainly responsible for the undesirable accumulation of the biogenic amines tyramine and putrescine in cheeses. The biosynthesis of tyramine and putrescine has been described as a species trait in Enterococcus faecalis. Tyramine is formed by the decarboxylation of the amino acid tyrosine, by the tyrosine decarboxylase (TDC) route encoded in the tdc cluster. Putrescine is formed from agmatine by the agmatine deiminase (AGDI) pathway encoded in the agdi cluster. These biosynthesis routes have been independently studied, tyrosine and agmatine transcriptionally regulate the $t d c$ and agdi clusters. The objective of the present work is to study the possible coregulation among TDC and AGDI pathways in E. faecalis. In the presence of agmatine, a positive correlation between putrescine biosynthesis and the tyrosine concentration was found. Transcriptome studies showed that tyrosine induces the transcription of putrescine biosynthesis genes and up-regulates pathways involved in cell growth. The tyrosine modulation over AGDI route was not observed in the mutant $\Delta t d c$ strain. Fluorescence analyses using gfp as reporter protein revealed PaguB (the promoter of agdi catabolic genes) was induced by tyrosine in the wild-type but not in the mutant strain, confirming that $t d c$ cluster was involved in the tyrosine induction of putrescine biosynthesis. This study also suggests that AguR (the transcriptional regulator of agdi) was implicated in interaction among the two clusters.

Keywords: Enterococcus faecalis, putrescine, tyramine, biogenic amines, regulation, biosynthesis

\section{INTRODUCTION}

Species belonging to Enterococcus genus are part of the cheeses microbiota, where they can reach $10^{5}$ to $10^{7}$ colony forming units (cfu) $\mathrm{g}^{-1}$ in the final product. These bacteria are present in the milk and are mainly found in traditional cheeses produced with raw milk. However, enterococci can also accumulate in cheeses elaborated with pasteurized milk as a consequence of contaminations during the fabrication course (Giraffa, 2003). Moreover, it has been observed that pasteurization does not completely eliminate them (Ladero et al., 2011).

Enterococci contribute to the cheese maturation, participating in the organoleptic properties development. Furthermore, they comprise interesting biotechnology features such as lipolytic activities, citrate utilization, volatile compounds biosynthesis, and bacteriocin production. Indeed, 
some Enterococcus faecalis strains with ability to produce bacteriocins have been proposed as adjunct cultures for food preserving (Giraffa, 2003).

Nevertheless, several authors have found a relation between the enterococci amounts in cheeses and the concentrations of tyramine (Burdychova and Komprda, 2007; Fernandez et al., 2007; Bonetta et al., 2008; Ladero et al., 2010b) and putrescine (Ladero et al., 2012a). Therefore, enterococci are considered mainly responsible for the undesirable accumulation of the biogenic amines (BAs) tyramine and putrescine in cheeses (Linares et al., 2012).

The consumption of foods with high concentrations of tyramine can cause intoxications. In fact, tyramine is responsible of the "cheese effect" (ten Brink et al., 1990) which involves symptoms as migraines and hypertension and can even cause cerebral hemorrhages (Ladero et al., 2010a; EFSA, 2011; Pessione, 2012). Tyramine citotoxicity has been recently demonstrated in vitro, indicating that at concentrations frequently encountered in BA-rich food, this BA produces necrosis in intestinal cells (Linares et al., 2016). In the same way, tyramine shows a cytotoxic synergistic effect with histamine - a common BA also found in cheeses (Del Rio et al., 2017). Although non-toxic effects have been directly related with diet putrescine, this BA could have a role in promoting some types of cancer (Alvarez and Moreno-Arribas, 2014) and is involved in the nitrosamines formation in foods, which have a well-known carcinogenic effect (Drabik-Markiewicz et al., 2011).

The production of unwanted BA has been studied in Enterococcus from food, human and clinical origin, identifying tyramine or putrescine producing strains among different species (Ladero et al., 2009, 2012b; Jimenez et al., 2013). Furthermore, the biosynthesis of tyramine and putrescine has been described as a species level trait in E. faecalis (Ladero et al., 2012b).

Tyramine is formed by the decarboxylation of the amino acid tyrosine, which exerts a role in the maintaining of the $\mathrm{pH}$ homeostasis in E. faecalis (Perez et al., 2016). The tyrosine decarboxylase (TDC) route is encoded in the $t d c$ cluster, which comprises four genes (Figure 1). The catabolic genes $t d c A$, tyrP, and $n h a C-2$ are co-transcribed as a polycistronic mRNA which expression is induced by tyrosine concentrations and acidic $\mathrm{pH}$ (Perez et al., 2015).

In E. faecalis, putrescine is formed from agmatine by the agmatine deiminase (AGDI) pathway, which is repressed by carbon source, suggesting a role in the energy production (Suarez et al., 2013). Five genes grouped in the agdi cluster are responsible of its biosynthesis: the regulator gene $a g u R$ and the metabolic genes $a g u B, a g u D, a g u A$, and $a g u C$ (Figure 1). $a g u R$ is constitutively transcribed as a monoscistronic mRNA from its promoter PaguR in a very low expression level, while the catabolic genes are co-transcribed in a single mRNA from the $a g u B$ promoter $(\mathrm{PaguB})$ in a divergent orientation (Linares et al., 2014). Furthermore, increasing concentrations of the substrate, agmatine, increase the transcription of the catabolic genes through the transmembrane regulator AguR (Suarez et al., 2013; Linares et al., 2014). Linares et al. (2015) suggest that AguR is a one component transcriptional activator that senses agmatine

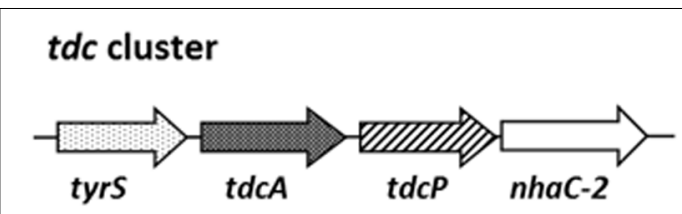

\section{agdi cluster}
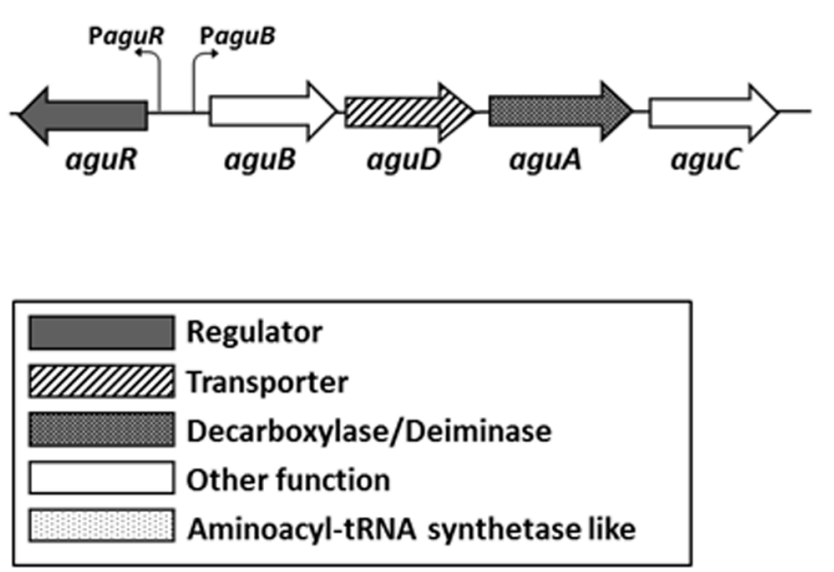

FIGURE 1 | Genetic organization of the tyrosine decarboxylase $(t d c)$ and agmatine deiminase (agdi) clusters in Enterococcus faecalis. The promoters of the aguR gene and aguBDAC operon are indicated. Adapted from Linares et al. (2014) and Ladero et al. (2017).

concentrations in the extracellular environment activating the transcription of the aguBDAC operon through its interaction with $\mathrm{PaguB}$.

Although both E. faecalis BA biosynthesis routes have been extensively studied independently, it is unknown whether there is a relationship between them. Therefore, the objective of this work was to examine the potential co-regulation among TDC and AGDI catabolic pathways in E. faecalis. First, we considered whether the amino acid substrate of one route had any effect on the other. Once it was verified that in the presence of tyrosine, the biosynthesis of putrescine increased, we studied the responsible mechanisms of this putative modulation through a global analysis of the gene expression in the presence and absence of tyrosine, and the putative role of the genes involved in the biosynthesis of these BA.

\section{MATERIALS AND METHODS}

\section{Bacterial Strains and Growth Conditions}

Two strains were used in this study: the wild-type E. faecalis V583 (Sahm et al., 1989) (hereafter referred to as wt) which biosynthesizes tyramine and putrescine (Ladero et al., 2012b) and the derived non-tyramine-producing mutant E. faecalis V583 $\Delta t d c$ (hereafter referred to as $\Delta t d c$ ) that lacks tyrosine 
decarboxylase genes cluster (Perez et al., 2015). Bacteria were grown in M17 medium (Oxoid, Hampshire, United Kingdom) supplemented with $5 \mathrm{~g} \mathrm{~L} \mathrm{~L}^{-1}$ glucose (Merck, Darmstadt, Germany) (GM17) at $37^{\circ} \mathrm{C}$ under aerobic conditions. Tyrosine, agmatine, or tyramine (Sigma-Aldrich, St. Louis, MO, United States) were added at indicated concentrations. Chloramphenicol $\left(5 \mu \mathrm{g} \mathrm{ml}^{-1}\right)$ (Sigma-Aldrich) was added when required. All cultures were inoculated at $0.1 \%(\mathrm{v} / \mathrm{v})$.

\section{Plasmid and Bacteria Transformation}

The agmatine-inducible plasmid pAGEnt-GFP (Figure 2) carries $\mathrm{PaguR}$, the aguR gene, and $\mathrm{PaguB}$ of E. faecalis V583 cloned to the promoterless gene $g f p$ encoding green fluorescent protein (GFP) (Linares et al., 2014). pAGEnt-GFP was transformed into electrocompetent $E$. faecalis cells obtained as described previously (Perez et al., 2015).

\section{Quantification of Biogenic Amines}

Supernatants obtained from centrifuged cultures were filtered through $\quad 0.2 \mu \mathrm{m}$ polytetrafluoroethylene (PTFE) filters (VWR, Barcelona, Spain). BA were derivatized with diethyl ethoxymethylenemalonate (DEEMM) (Sigma-Aldrich). $100 \mu \mathrm{l}$ of sample were mixed with $175 \mu \mathrm{l}$ of $1 \mathrm{M}$ borate buffer (1 M boric acid neutralized with $\mathrm{NaOH}$ until $\mathrm{pH} 9.0), 75 \mu \mathrm{l}$ of methanol (Merck), $2 \mu \mathrm{l}$ of L-2-aminoadipic acid as internal standard (2 g/L) (Sigma-Aldrich) and $3 \mu \mathrm{l}$ of DEEMM. The mixture was incubated at $30^{\circ} \mathrm{C}$ in an ultrasound bath (Selecta, Barcelona, Spain) for $45 \mathrm{~min}$. Samples were then heated at $70^{\circ} \mathrm{C}$ for $2 \mathrm{~h}$ to allow the complete degradation of excess DEEMM and reagent by-products. Samples were filtered through $0.2 \mu \mathrm{m}$ PTFE membranes (VWR) before injection into the chromatograph system. Samples were

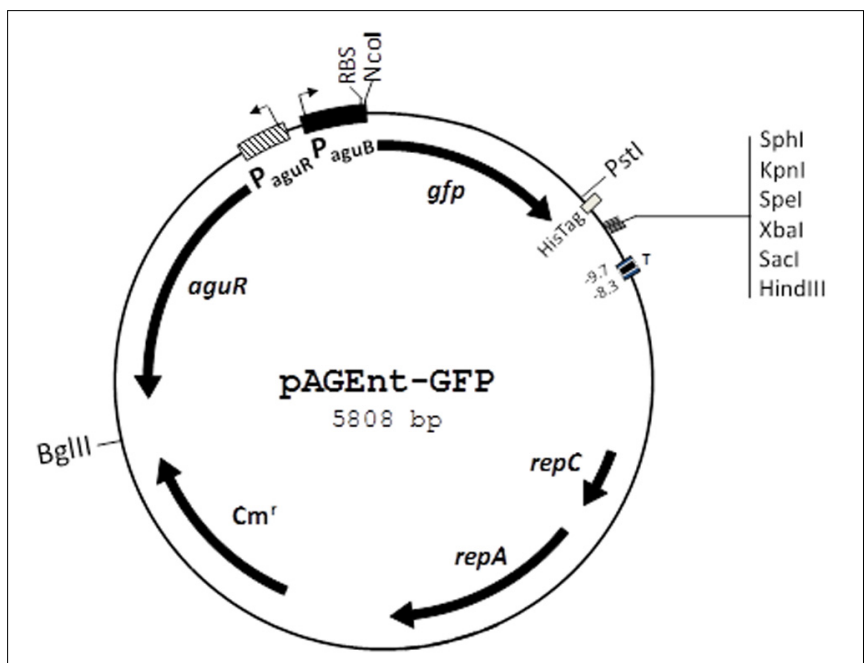

FIGURE 2 | Genetic map of the pAGEnt-GFP vector. repC and repA, replication genes; $\mathrm{Cm}^{r}$, chloramphenicol resistance gene; aguR, gene encoding the regulatory protein AguR; PaguR, aguR promoter; PaguB, aguBDAC promoter; RBS, ribosome binding site; gfp, gene encoding green fluorescent protein; His-Tag, C-terminal histidine tag; T, transcription terminator. Restriction sites are indicated. Adapted from Linares et al. (2014). diluted, when necessary, with $0.1 \mathrm{~N} \mathrm{HCl}$ (Merck). BA were separated and quantified by ultra high performance liquid chromatography (UHPLC) system (Waters, Milford, MA, United States) with an $\mathrm{UPLC}^{\circledR} \mathrm{BEH}$ C18 $1.7 \mu \mathrm{m}$ column (Waters), following the method previously described (Redruello et al., 2013). Empower 2 software (Waters) was used to control the system and to analyze the data. Standards were prepared with agmatine, putrescine dihydrochloride (Acros Organics, Geel, Belgium), tyrosine and tyramine in Milli-Q water. The BA concentrations provided are the average of three independent cultures.

\section{DNA Microarrays and Data Analysis}

DNA microarrays of E. faecalis V583 (Agilent Technologies, Santa Clara, CA, United States) were designed using the Agilent eArray (v5.0) program according to the manufacturer's instructions. Each microarray $(8 \mathrm{~K} \times 15 \mathrm{~K})$ was designed to contain spots of two different 60-mer oligonucleotide probes (in duplicate) specific for each of the 3182 coding DNA sequences (CDSs) representing the chromosomal genes of the E. faecalis V583 genome (GenBank accession no. AE016830) (Paulsen et al., 2003). The design and disposition of the probes in the array were deposited in Gene Expression Omnibus (GEO) database (Platform GPL21449).

Total RNA was isolated from $30 \mathrm{ml}$ of E. faecalis wt strain cultures grown in GM17 supplemented with $5 \mathrm{mM}$ agmatine and in the presence or absence of $15 \mathrm{mM}$ tyrosine until the end of the exponential phase of growth $(7 \mathrm{~h})$, as described by Perez et al. (2016). cDNA was synthesized from $20 \mu \mathrm{g}$ of RNA using the SuperScript III reverse transcriptase kit (Life Technologies, Bleiswijk, The Netherlands). Then, $20 \mu \mathrm{g}$ of cDNA were labeled with DyLight 550 or DyLight 650 dyes using the DyLight amine-reactive dyes kit (Thermo Scientific, Amsterdam, Netherlands). 300 ng of DyLight 550and $300 \mathrm{ng}$ of DyLight 650-labeled cDNA were mixed and hybridized for $17 \mathrm{~h}$ at $60^{\circ} \mathrm{C}$ in the E. faecalis V583 DNA microarray using the In situ Hybridization Kit Plus, the Hybridization Gasket Slide and the Agilent G2534 A Microarray Hybridization Chamber (Agilent Technologies) following the manufacturer's indications, as previously described (Perez et al., 2016). Slides were scanned using a GenePix 4200 A Microarray Scanner (Molecular Devices, Sunnyvale, CA, United States) and images were analyzed using GenePix Pro v.6.0 software. Background subtraction and locally weighted scatterplot smoothing normalization were done using the standard routines provided by GENOME2D software available at http://genome2d.molgenrug.nl/index.php/analysispipeline. DNA microarray data were obtained from two independent biological replicates and one technical replicate (including a dye swap). Expression ratios were calculated from the comparison of four spots per gene per microarray (total of 20 measurements per gene). A gene was considered differentially expressed when a $p$-value of at least 0.001 was obtained and the expression fold-change was at least $|2|$. The microarray data were deposited in GEO database under the Accession no. GSE97219. Functional analysis of the genes differentially expressed was performed using Gene Set Enrichment 
Analysis (GSEA) using routines provided by GENOME2D software available at http://server.molgenrug.nl/index.php/ gsea-pro.

\section{Quantification of Gene Expression by Reverse Transcription Quantitative PCR (RT-qPCR)}

Gene expression of aguA gene was measured by RT-qPCR in cultures of the strains wt and $\Delta t d c$. First, bacteria from cultures adjusted to a cell density of approximately $2\left(\mathrm{Abs}_{600}\right)$ were harvested by centrifugation at $4^{\circ} \mathrm{C}$ and total RNA was extracted using TRI reagent (Sigma-Aldrich) as previously described (Linares et al., 2009). RNA samples (2 $\mu \mathrm{g}$ ) were treated with $2 \mathrm{U}$ of DNase I (Fermentas, Madrid, Spain) for $2 \mathrm{~h}$, in order to eliminate any genomic contamination. Total cDNA was synthesized from $0.5 \mu \mathrm{g}$ of RNA using the reverse transcription iScript ${ }^{\mathrm{TM}}$ cDNA Synthesis kit (Bio-Rad Madrid, Spain). Gene expression was performed in a 7500 Fast real-time PCR System (Applied Biosystems, Carlsbad, CA, United States). After fourfold dilution of cDNA sample, $5 \mu \mathrm{l}$ were mixed with $12.5 \mu \mathrm{l}$ of SYBR Green PCR Master Mix (Applied Biosystems), $1 \mu \mathrm{l}$ of each primer at $700 \mathrm{nM}$ and $5.5 \mu \mathrm{l}$ of RNase-free water. The primers aguAqF and aguAqR (Linares et al., 2014), which are specific for aguA gene of $E$. faecalis, were used. The expression of $\operatorname{rec} A$ (the recombinase A encoding gene) and tufA (the elongation factor thermo-unstable encoding gene) were used as internal controls to normalize the RNA concentration, using the specific primers recAF and recAR, and EFV583-tufF and EFV583-tufR, respectively (Linares et al., 2014). Amplifications were performed using the default cycling settings established by Applied Biosystems. Gene expression was calculated following the $2^{-\Delta \Delta C_{T}}$ method (Livak and Schmittgen, 2001). RT-qPCR analysis was performed on RNA purified from three biological replicates for each condition.

\section{Green Fluorescence Measurements}

Total fluorescence of wt and $\Delta t d c$ cells harboring pAGEntGFP vector was measured following previous indications (Linares et al., 2014). Briefly, cells from cultures adjusted to a cell density $2\left(\mathrm{Abs}_{600}\right)$ were harvested, washed in $400 \mu \mathrm{l}$ of $50 \mathrm{mM}$ potassium phosphate buffer $\mathrm{pH} 7.2$ and resuspended in $200 \mu \mathrm{l}$ of the same buffer. GFP emission was determined using a Cary Eclipse fluorescence spectrophotometer (Varian, Inc., Palo Alto, CA, United States), at 485 and $530 \mathrm{~nm}$ of excitation and emission wavelengths, respectively. Background fluorescence levels were assessed by measuring non-fluorescent control cells and subtracting the obtained values.

\section{Statistical Analysis}

Results were presented as means and standard deviations. Statistical analyses were performed with the software SPSS version 15.0 (SPSS, Inc., Chicago, IL, United States) using the Student's $t$-test, ANOVA and the Tukey post hoc test as indicated. Significance was set at $p<0.05$.

\section{RESULTS}

\section{The Production of Putrescine Increases with Tyrosine Concentration}

The effect of the tyrosine (tyramine substrate) concentration of culture medium on the putrescine biosynthesis was studied by growing the wt strain in GM17 supplemented with $5 \mathrm{mM}$ agmatine (putrescine substrate) and $0,5,10$, and $15 \mathrm{mM}$ tyrosine until the end of the exponential phase of growth $(t=7 \mathrm{~h})$. Then, putrescine concentration was measured by UHPLC. As shown in Figure 3, putrescine biosynthesis correlated positively with the tyrosine concentration.

We also investigated the influence of agmatine concentration in tyramine biosynthesis. However, no significant differences were found (data not shown), suggesting no correlation between agmatine concentration and tyramine biosynthesis.

\section{Transcriptomic Studies Show an Induction of the Putrescine Biosynthesis Genes in the Presence of Tyrosine}

To seek for genes that could be involved in the observed increase of putrescine production in the presence of tyrosine, DNA microarrays were performed. The transcriptomic profile of the wt strain grown with or without tyrosine was compared. The expression of 394 genes was significantly different, 277 being induced in presence of tyrosine. In total, $12.4 \%$ of chromosomal genes showed an altered expression. Those genes with different expression were classified by the Clusters of

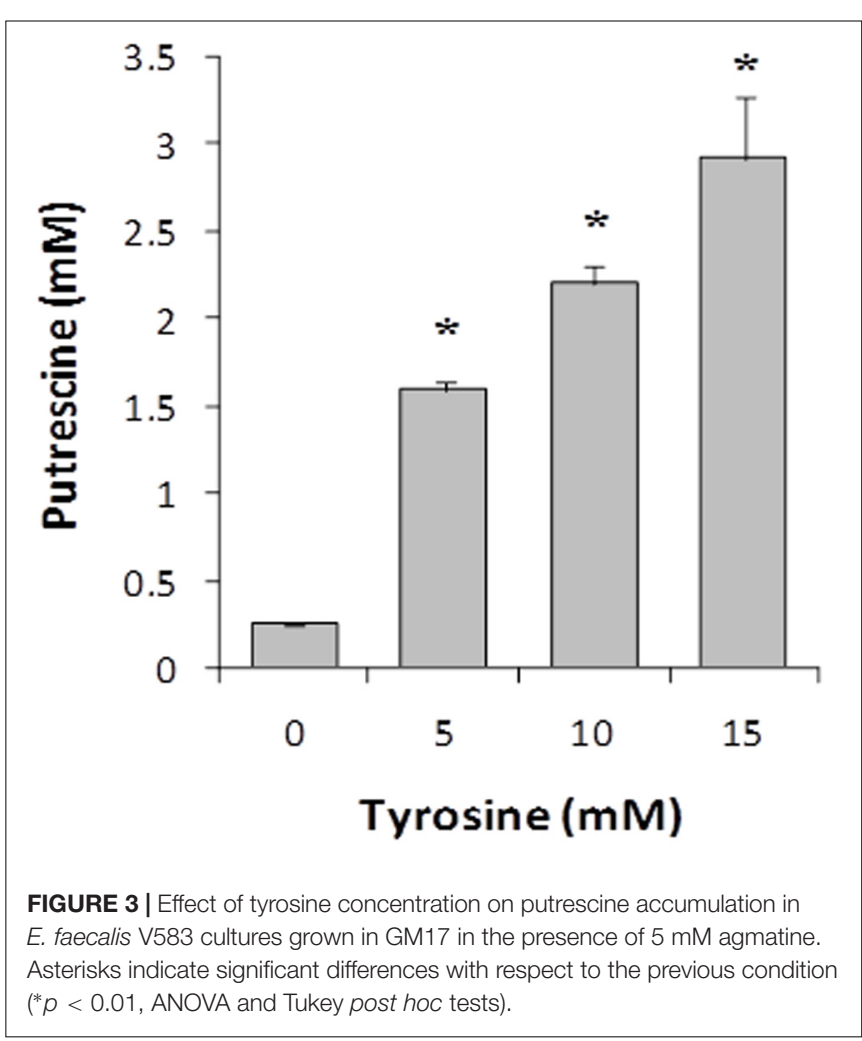




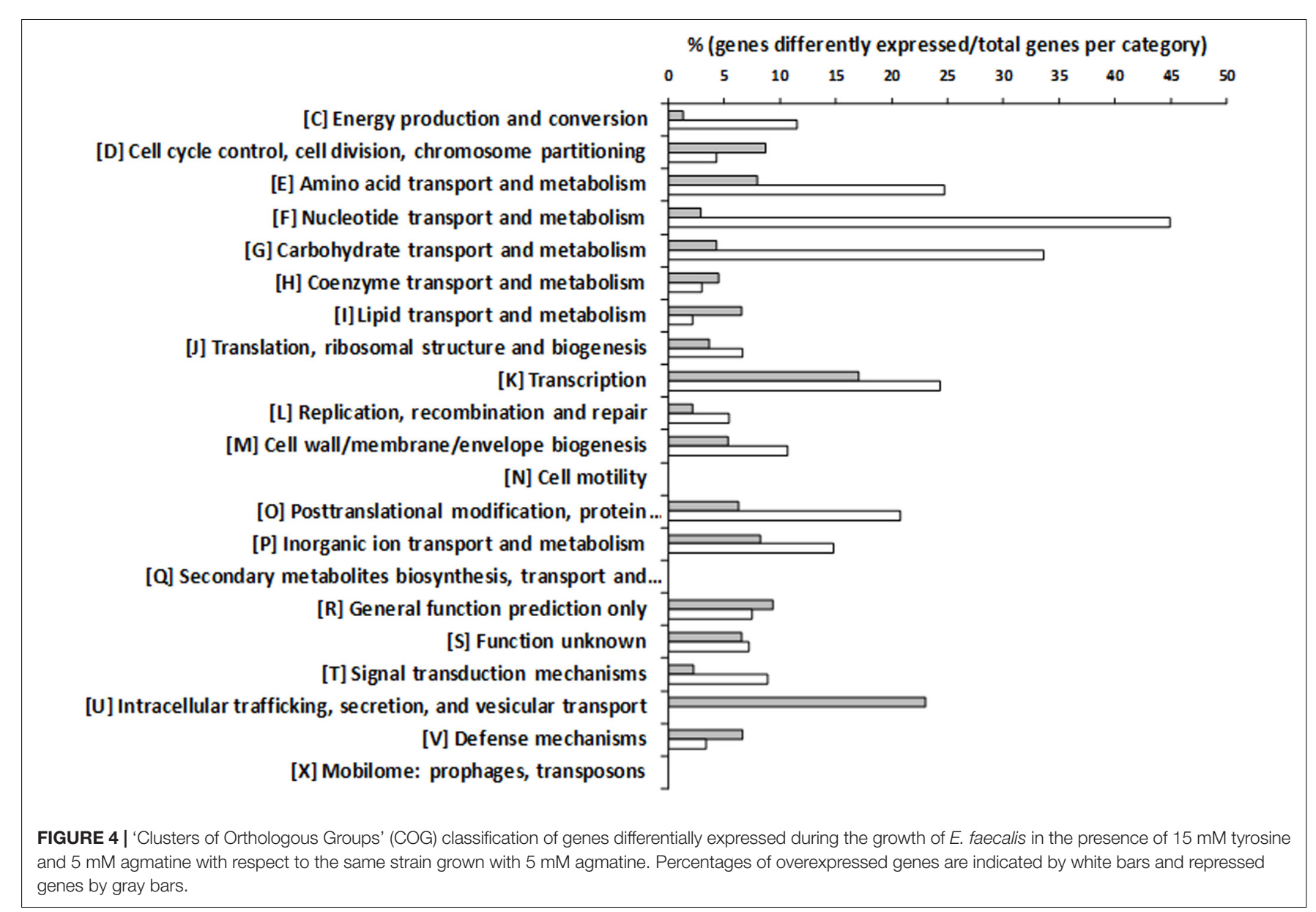

Orthologous Groups (COG) categories of the National Centre for Biotechnology Information (NCBI) ${ }^{1}$ (Supplementary Table S1) and their relevancies are shown in Figure 4. Most of the genes were assigned to the categories of nucleotides transport and metabolism, with the $48 \%$ of genes showing an altered expression, followed by carbohydrates (38\%) and amino acids (33\%) transport and metabolism categories. Furthermore, $41 \%$ of the genes assigned to transcription category showed a different expression.

As could be expected, the presence of tyrosine implied a repression of tyrosine biosynthesis, since most of the genes involved in tyrosine synthesis, including the shikimate pathway, were repressed (Supplementary Tables S1, S2 and Supplementary Figure S1).

Functional analysis of the differentially expressed genes made by GSEA, revealed the induction of pathways related with cell growth. Thus, it was observed an enhancement of purine and pyrimidine precursor supply routes related to DNA biosynthesis; an activation of aminosugars metabolism to provide precursors for cell wall biosynthesis; and an increment in the levels of aminoacyl-tRNA synthetases for protein biosynthesis (Supplementary Table S2 and Supplementary Figure S1).

${ }^{1}$ www.ncbi.nlm.nih.gov/COG/
Focusing on the genes involved in the BA biosynthesis, the expression of all the genes belonging to the $t d c$ and agdi clusters was significantly different (Table 1). As expected, when E. faecalis was grown in the presence of tyrosine, the metabolic genes implicated in tyramine biosynthesis $(t d c A, t d c P$, and $n h a c-2)$ were induced. Furthermore, the genes responsible of putrescine biosynthesis $(a g u B, a g u D, a g u A$, and $a g u C)$, were also induced in the presence of tyrosine.

\section{RT-qPCR Results Confirm That Putrescine Biosynthesis Genes Are Induced by Tyrosine}

To confirm the agdi cluster expression results obtained in the transcriptomic profile, we quantified by RT-qPCR the expression of $\operatorname{aguA}$ gene, as representative of the whole polycistronic aguBDAC mRNA. Total RNA was isolated from cultures of the wt strain grown with or without tyrosine, i.e., as they were cultivated for the microarray experiment.

The expression of aguA showed a significantly increase (ninefold) in the presence of tyrosine (Figure 5). In concordance with the previous results, putrescine biosynthesis reached about 10 -fold concentrations when the amino acid tyrosine was added to the medium. These results support that tyrosine induces the putrescine biosynthesis genes. 
TABLE 1 | Expression of the agdi and tdc cluster genes in the transcriptome of $E$. faecalis V583 grown with or without $15 \mathrm{mM}$ tyrosine.

\begin{tabular}{|c|c|c|c|c|c|}
\hline & Gene & Locus & Description & Fold change & $p$-Value \\
\hline \multirow[t]{5}{*}{ agdi cluster } & aguR & EF0731 & Transcriptional regulator & 2.48 & 6.81E-04 \\
\hline & aguB & EF0732 & Putrescine carbamoyltransferase & 8.05 & 5.24E-04 \\
\hline & $\operatorname{aguD}$ & EF0733 & Agmatine/putrescine antiporter & 6.88 & 4.18E-04 \\
\hline & $\operatorname{aguA}$ & EF0734 & Agmatine deiminase & 6.34 & 4.41E-04 \\
\hline & aguC & EF0735 & Carbamate kinase & 5.73 & 5.38E-04 \\
\hline \multirow[t]{4}{*}{ tdc cluster } & tyrs & EF0633 & Tyrosyl-tRNAsynthetase & -8.73 & $2.41 \mathrm{E}-04$ \\
\hline & $t d c A$ & EF0634 & Tyrosine decarboxylase & 3.23 & 2.45E-04 \\
\hline & $t d c P$ & EF0635 & Tyrosine/tyramine exchanger & 2.56 & 3.36E-04 \\
\hline & nhac-2 & EF0636 & $\mathrm{Na}^{+} / \mathrm{H}^{+}$antiporter & 2.21 & 6.04E-04 \\
\hline
\end{tabular}

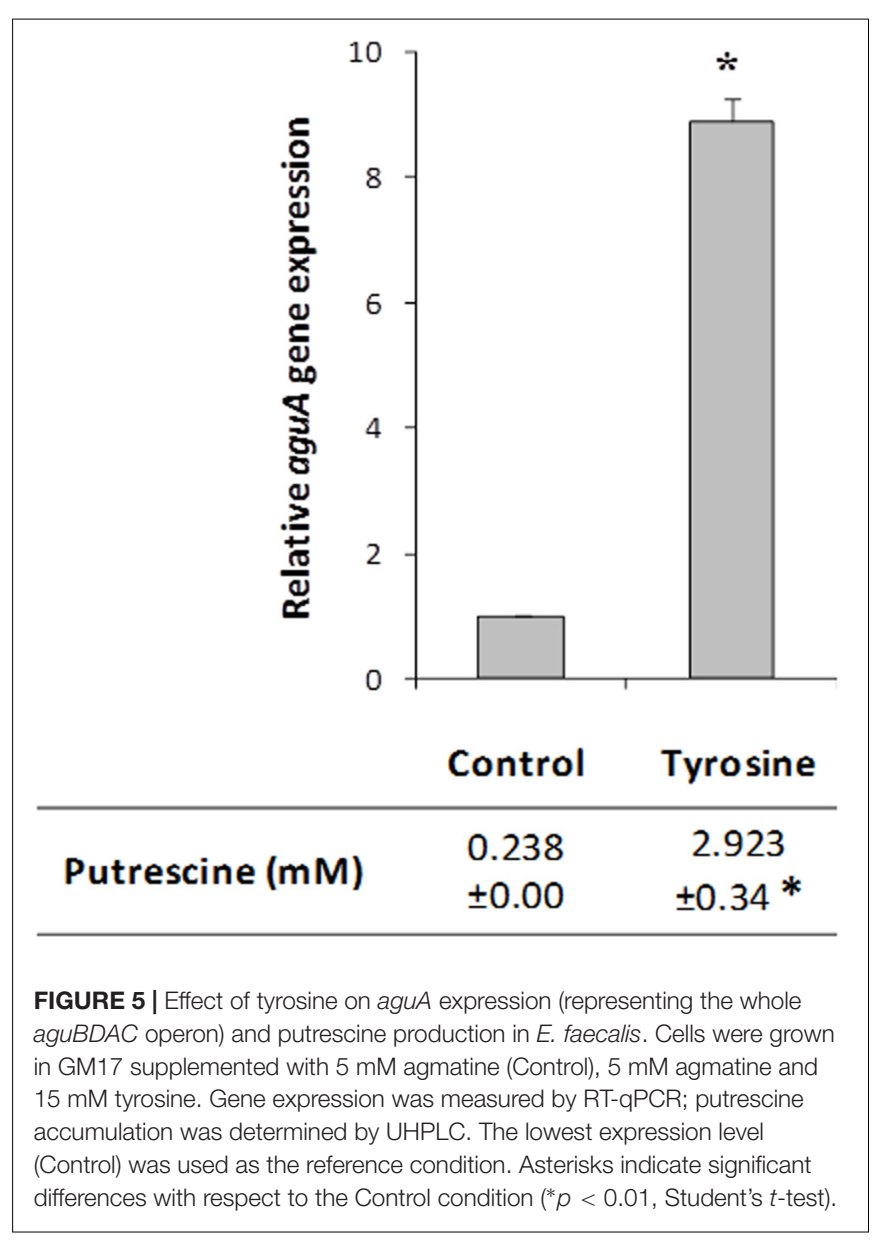

\section{The tdc Cluster Is Involved in the Tyrosine Induction of Putrescine Biosynthesis}

To test a potential role of the tyramine biosynthesis genes -the $t d c$ cluster-, aguA expression and putrescine production were measured in cultures of the $\Delta t d c$ mutant strain grown in presence or absence of tyrosine. Unlike the wt strain results, no differences were observed in the aguA expression or putrescine production when tyrosine was added (Figure 6). This could be due to the fact that the product of the reaction, the tyramine - which is not

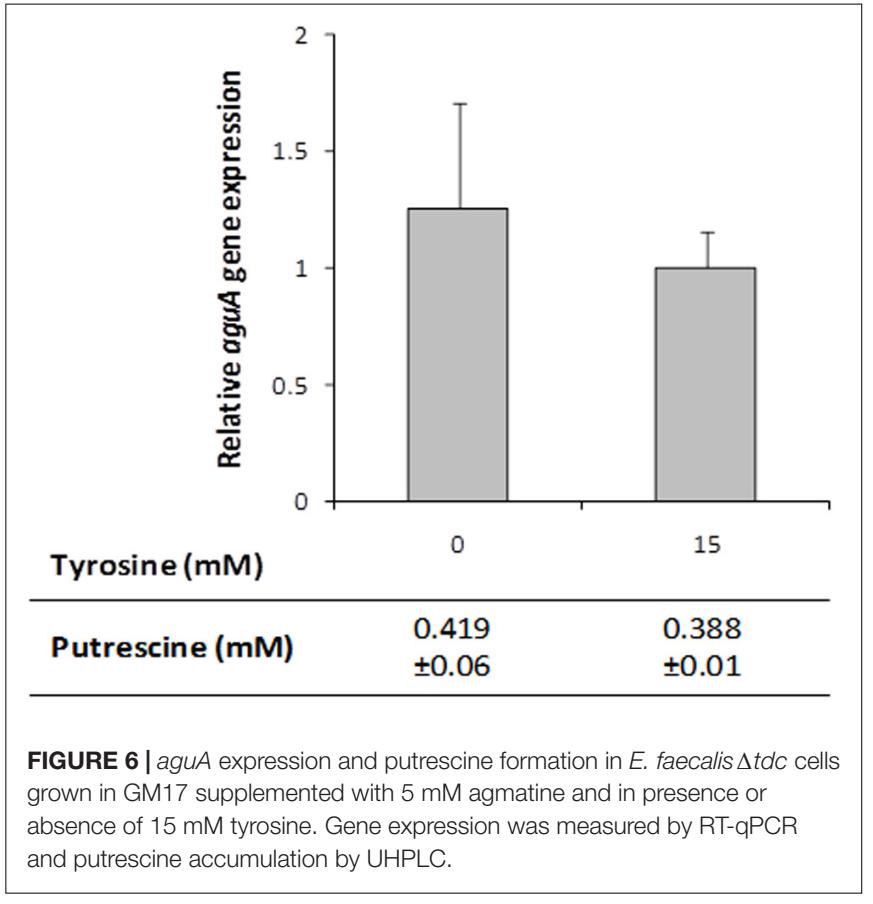

produced in the $\Delta t d c$ mutant - was the inducer. To investigate it, the wt strain was grown with $5 \mathrm{mM}$ agmatine and with and without $15 \mathrm{mM}$ tyramine. No variation in $a g u A$ expression or putrescine production was observed when tyramine was present in the culture, indicating that it was not responsible of putrescine biosynthesis genes induction (data not shown).

\section{The Role of aguR-PaguB and tdc Cluster in the Induction by Tyrosine}

To further study the tyrosine induction, we used the pAGEntGFP construct (Figure 2), in which the $g f p$ gene is under the control of the $a g u R-\mathrm{PaguB}$ cassette. The wt and $\Delta t d c$ strains harboring pAGEnt-GFP were grown for $8 \mathrm{~h}$ in medium supplemented with $5 \mathrm{mM}$ agmatine and $0,5,10$, or $15 \mathrm{mM}$ tyrosine. Fluorescence measurements in the wt strain revealed that $g f p$ expression correlated positively with the tyrosine concentration, reaching up to twofold units in presence of $10 \mathrm{mM}$ tyrosine (Figure 7A). Conversely, no significantly differences 

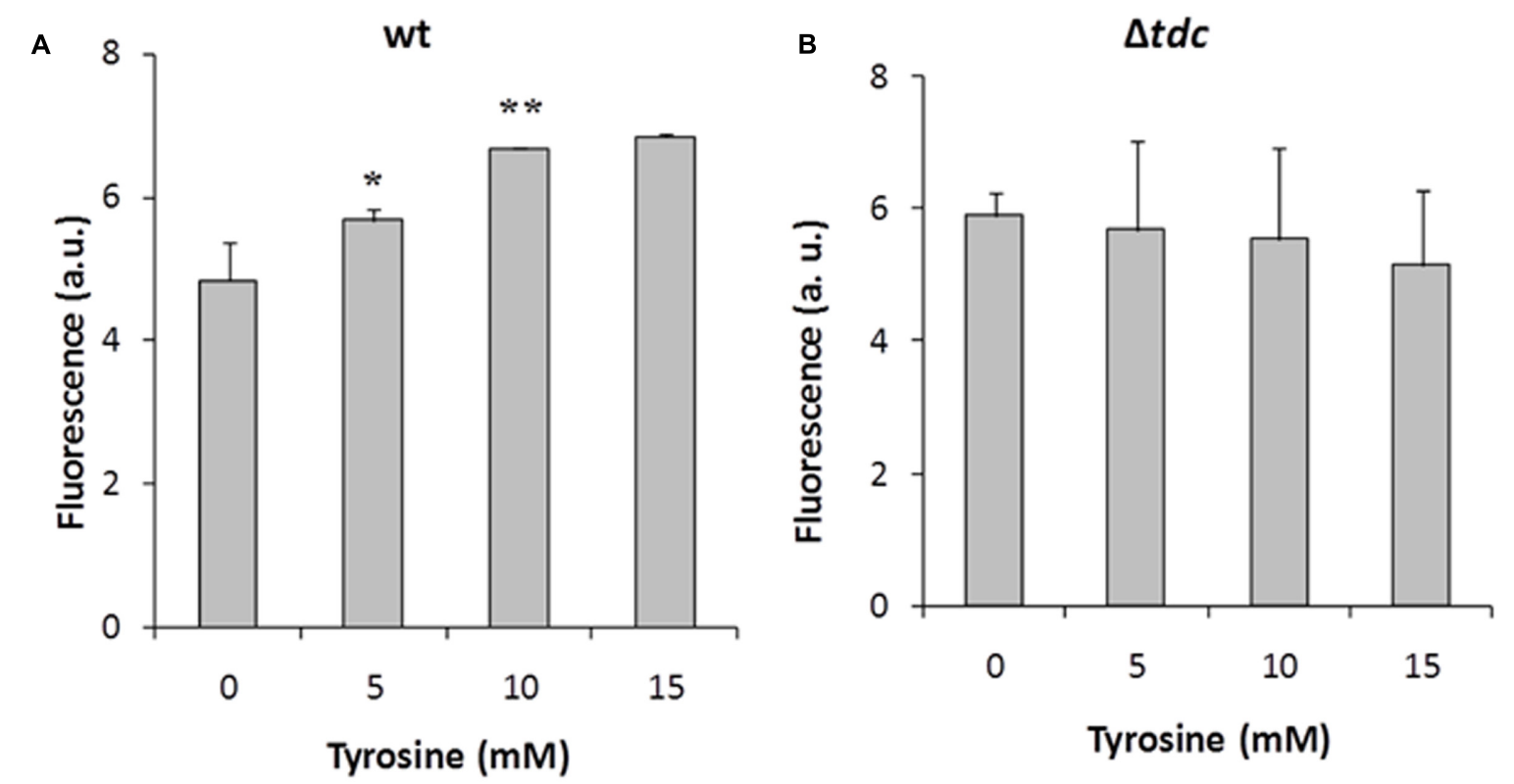

FIGURE 7 | Induction of gfp in E. faecalis wt (A) and $\Delta t d c$ (B) cultures containing pAGEnt-GFP in the presence of $5 \mathrm{mM}$ agmatine and under a range of tyrosine concentrations. Fluorescence was measured after $8 \mathrm{~h}$ of culture. Asterisks indicate significant differences with respect to the previous condition ${ }^{*} p<0.05$, ${ }^{* *} p<0.01$, ANOVA and Tukey post hoc tests). a.u., arbitrary units.

were observed in the fluorescence signal of $\Delta t d c$ cultures (Figure 7B).

Hence, the protein AguR and the $a g u B$ promoter are both implicated in the regulation of the aguBDAC genes transcription mediated by the $t d c$ cluster in the presence of tyrosine.

\section{DISCUSSION}

Previous studies in E. faecalis have shown that the genes responsible for tyrosine and putrescine biosynthesis are induced by their respective substrates, tyrosine and agmatine (Linares et al., 2014; Perez et al., 2015). Although the genetic regulation of different BA biosynthesis pathways in different microorganisms has been studied, possible co-regulation between different BA pathways within one bacterium has not been widely described. Since E. faecalis produces tyramine and putrescine, we examined the potential co-regulation among TDC and AGDI pathways, studying in principle whether the substrates have any effect on the other metabolic pathway. In fact, the higher the concentration of tyrosine, the greater the production of putrescine (Figure 3). Nevertheless, this effect was not reciprocal, since the production of tyramine remained constant independently of agmatine concentration.

A reciprocal association between tyramine and putrescine biosynthesis has been suggested in Lactobacillus brevis (Russo et al., 2012) showing an induction of both pathways when both precursors were present. In contrast, other authors observed the repression of the arginine deiminase pathway by histidine decarboxylase route in Lactobacillus hilgardii (Lamberti et al., 2011) and by glutamate decarboxylase pathway in Lactococcus lactis (Mazzoli et al., 2010) and Lactobacillus reuteri (Teixeira et al., 2014).

To deepen the genetic regulation, we further investigated the tyrosine effect on the transcriptome of E. faecalis. The $t d c$ cluster transcription observed in the microarray experiment (Table 1) concurs with our previous results obtained by RT-qPCR when the effect of the substrate amino acid on $t d c$ cluster expression was assessed (Perez et al., 2015). I.e., the catabolic genes $t d c A, t d c P$, and nhac- 2 - that encode a tyrosine decarboxylase, a tyrosinetyramine antiporter and a $\mathrm{Na}^{+} / \mathrm{H}^{+}$antiporter, respectively are over-expressed by tyrosine, while the tyrS which encodes an aminoacyl-tRNA synthetase-like gene is repressed. This effect of tyrosine on $t d c$ cluster expression has also been demonstrated in other E. faecalis strains by Bargossi et al. (2015, 2017).

Focusing on the putrescine biosynthesis genes, the global transcriptome analysis proved that tyrosine induces the catabolic genes of the agdi cluster (Table 1). The induction of the agdi catabolic operon by tyrosine was ascertained by RT-qPCR analysis of $a g u A$ expression, showing a rise of ninefold in the presence of tyrosine (Figure 5). Tyrosine induction of putrescine biosynthesis genes was not observed in the mutant strain $\Delta t d c$ (Figure 7B), which is unable to decarboxylate tyrosine and produce tyramine (Perez et al., 2015). Therefore, the product of the reaction, tyramine, could be the inducer. However, we found that tyramine had no effect on aguA expression or putrescine biosynthesis in the wt strain. Landete et al. (2007) also observed that tyramine does not affect putrescine production in other $E$. faecalis strains. In addition to the cluster $t d c$ was necessary, the use of the $g f p$ reporter gene established that tyrosine induction of the agdi cluster was performed throughout the aguR-PaguB cassette (Figure 7A). The phenotypic differences observed are 
smaller than those observed in transcriptome and RT-qPCR experiments. This could be explained by the lower sensitivity of the whole-cell fluorescence technique, and the contrast between the agdi cluster, which is located in the chromosome and the reporter gene located in a multicopy plasmid, which implies a greater background. The use of $g f p$ also verified that the PaguB promoter is not induced by tyrosine in the mutant strain $\Delta t d c$ (Figure 7B).

Further analysis need to be done in order to investigate the mechanism of the induction exerted by tyrosine and the $t d c$ cluster on the AGDI pathway. Since the transcriptional regulator AguR is a transmembrane protein (Linares et al., 2015), the antiporter TdcP or the tyrosine decarboxylase TdcA - which are membrane proteins (Pessione et al., 2009) - could interact with AguR enhancing its union to $a g u B$ promoter and inducing the expression of $a g u B D A C$.

Tyrosine - besides being a BA precursor that induces the $t d c$ transcription- could even work as an indicator of amino acids abundance for the bacterial cell and direct metabolism toward cell growth. In fact, functional analysis of the transcriptomic data indicated that a tyrosine excess inhibits its own biosynthesis route and activates pathways related to accumulation of precursors for DNA, glucolytic and pyruvate metabolism enzymes and cell wall biosynthesis. Moreover, an increment in the transcription of the genes encoding some aminoacyl-tRNA synthetases was also observed, suggesting the cell would be loading the corresponding tRNAs for protein biosynthesis. Supplementation with amino acids has been shown to act as a signal for growth in Bacillus subtilis by reducing the expression of genes related to amino acid biosynthesis and inducing genes involved in nucleotide metabolism (Mader et al., 2002; Pessione et al., 2009).

\section{CONCLUSION}

Tyrosine, which is the substrate of tyramine biosynthesis, is also an inducer of putrescine production in E. faecalis, the main producer of both BA in cheese. Moreover, the presence of tyrosine would be received by the enterococci cells as a signal to growth, what would lead in an increment in the number of

\section{REFERENCES}

Alvarez, M. A., and Moreno-Arribas, M. V. (2014). The problem of biogenic amines in fermented foods and the use of potential biogenic amine-degrading microorganisms as a solution. Trends Food Sci. Tech. 39, 146-155. doi: 10.1016/ j.tifs.2014.07.007

Bargossi, E., Tabanelli, G., Montanari, C., Gatto, V., Chinnici, F., Gardini, F., et al. (2017). Growth, biogenic amine production and tyrDC transcription of Enterococcus faecalis in synthetic medium containing defined amino acid concentrations. J. Appl. Microbiol. 122, 1078-1091. doi: 10.1111/jam.13406

Bargossi, E., Tabanelli, G., Montanari, C., Lanciotti, R., Gatto, V., Gardini, F., et al. (2015). Tyrosine decarboxylase activity of enterococci grown in media with different nutritional potential: tyramine and 2-phenylethylamine accumulation and tyrDC gene expression. Front. Microbiol. 10:259. doi: 10.3389/fmicb.2015. 00259

Bonetta, S., Carraro, E., Coisson, J. D., Travaglia, F., and Arlorio, M. (2008). Detection of biogenic amine producer bacteria in a typical Italian goat cheese. J. Food Prot. 71, 205-209. doi: 10.1021/jf8000586
BA-producing cells. Therefore, tyrosine appears to be a relevant amino acid in cheese, in the sense that it would increase the risk of accumulating tyramine and putrescine, which are health threatening for consumers.

\section{AUTHOR CONTRIBUTIONS}

MP carried out most of experiments, analyzed data and drafted the manuscript, VL analyzed some of the data and help to write the manuscript; BdR and BR performed some experiments; AdJ, OK, JK, MM, and MF participated in study design and data interpretation; AdJ, OK, and JK supervised the arrays experiments; MA provided the general concept, participated in study design and data interpretation, and supervised the work and the manuscript. All authors contributed to the discussion of the research and approved the final manuscript.

\section{FUNDING}

This work was performed with the financial support of the Spanish Ministry of Economy and Competitiveness (AGL201345431-R and AGL2016-78708-R), and via the GRUPIN14-137 project (the latter is co-financed by the Plan for Science, Technology and Innovation of the Principality of Asturias 20142017 and European Regional Development Funds).

\section{ACKNOWLEDGMENT}

The authors thank Adrian Burton for language and editing assistance.

\section{SUPPLEMENTARY MATERIAL}

The Supplementary Material for this article can be found online at: https://www.frontiersin.org/articles/10.3389/fmicb. 2017.02107/full\#supplementary-material

Burdychova, R., and Komprda, T. (2007). Biogenic amine-forming microbial communities in cheese. FEMS Microbiol. Lett. 276, 149-155. doi: 10.1111/j. 1574-6968.2007.00922.x

Del Rio, B., Redruello, B., Linares, D. M., Ladero, V., Fernandez, M., Martin, M. C., et al. (2017). The dietary biogenic amines tyramine and histamine show synergistic toxicity towards intestinal cells in culture. Food Chem. 218, 249-255. doi: 10.1016/j.foodchem.2016. 09.046

Drabik-Markiewicz, G., Dejaegher, B., De Mey, E., Kowalska, T., Paelinck, H., and Vander Heyden, Y. (2011). Influence of putrescine, cadaverine, spermidine or spermine on the formation of $\mathrm{N}$-nitrosamine in heated cured pork meat. Food Chem. 126, 1539-1545. doi: 10.1016/j.foodchem.2010.11.149

EFSA (2011). Scientific opinion on risk based control of biogenic amine formation in fermented foods. EFSA J. 9, 2393-2486.

Fernandez, M., Linares, D. M., Del Rio, B., Ladero, V., and Alvarez, M. A. (2007). HPLC quantification of biogenic amines in cheeses: correlation with PCRdetection of tyramine-producing microorganisms. J. Dairy Res. 74, 276-282. doi: $10.1017 /$ S0022029907002488 
Giraffa, G. (2003). Functionality of enterococci in dairy products. Int. J. Food Microbiol. 88, 215-222. doi: 10.1016/S0168-1605(03)00183-1

Jimenez, E., Ladero, V., Chico, I., Maldonado-Barragan, A., Lopez, M., Martin, V., et al. (2013). Antibiotic resistance, virulence determinants and production of biogenic amines among enterococci from ovine, feline, canine, porcine and human milk. BMC Microbiol. 13:288. doi: 10.1186/1471-2180-13-288

Ladero, V., Calles-Enríquez, M., Fernández, M., and Alvarez, M. A. (2010a). Toxicological effects of dietary biogenic amines. Curr. Nutr. Food Sci. 6, 145-156. doi: 10.2174/157340110791233256

Ladero, V., Canedo, E., Perez, M., Cruz Martin, M., Fernandez, M., and Alvarez, M. A. (2012a). Multiplex qPCR for the detection and quantification of putrescine-producing lactic acid bacteria in dairy products. Food Control 27, 307-313. doi: 10.1016/j.foodcont.2012.03.024

Ladero, V., Fernandez, M., Calles-Enriquez, M., Sanchez-Llana, E., Canedo, E., Martin, M. C., et al. (2012b). Is the production of the biogenic amines tyramine and putrescine a species-level trait in enterococci? Food Microbiol. 30, 132-138. doi: $10.1016 /$ j.fm.2011.12.016

Ladero, V., Fernandez, M., and Alvarez, M. A. (2009). Isolation and identification of tyramine-producing enterococci from human fecal samples. Can. J. Microbiol. 55, 215-218. doi: 10.1139/w08-133

Ladero, V., Linares, D. M., Pérez, M., del Rio, B., Fernández, M., and Alvarez, M. A. (2017). "Biogenic amines in dairy products," in Microbial Toxins in Dairy Products, ed. A. Y. Tamime (Chichester: John Wiley \& Sons, Ltd.), 94-131.

Ladero, V., Martinez, N., Martin, M. C., Fernandez, M., and Alvarez, M. A. (2010b). qPCR for quantitative detection of tyramine-producing bacteria in dairy products. Food Res. Int. 43, 289-295. doi: 10.1016/j.fm.2010.05.026

Ladero, V., Sanchez-Llana, E., Fernandez, M., and Alvarez, M. A. (2011). Survival of biogenic amine-producing dairy LAB strains at pasteurisation conditions. Int. J. Food Sci. Tech. 46, 516-521. doi: 10.1111/j.1365-2621.2010.02508.x

Lamberti, C., Purrotti, M., Mazzoli, R., Fattori, P., Barello, C., Coisson, J. D., et al. (2011). ADI pathway and histidine decarboxylation are reciprocally regulated in Lactobacillus hilgardii ISE 5211: proteomic evidence. Amino Acids 41, 517-527. doi: 10.1007/s00726-010-0781-2

Landete, J. M., Pardo, I., and Ferrer, S. (2007). Tyramine and phenylethylamine production among lactic acid bacteria isolated from wine. Int. J. Food Microbiol. 115, 364-368. doi: 10.1016/j.ijfoodmicro.2006.10.051

Linares, D. M., del Rio, B., Ladero, V., Martinez, N., Fernandez, M., Martin, M. C., et al. (2012). Factors influencing biogenic amines accumulation in dairy products. Front. Microbiol. 3:180. doi: 10.3389/fmicb.2012.00180

Linares, D. M., del Rio, B., Redruello, B., Ladero, V., Martin, M. C., de Jong, A., et al. (2015). AguR, a transmembrane transcription activator of the putrescine biosynthesis operon in Lactococcus lactis, acts in response to the agmatine concentration. Appl. Environ. Microbiol. 81, 6145-6157. doi: 10.1128/AEM. 00959-15

Linares, D. M., del Rio, B., Redruello, B., Ladero, V., Martin, M. C., Fernandez, M., et al. (2016). Comparative analysis of the in vitro cytotoxicity of the dietary biogenic amines tyramine and histamine. Food Chem. 197, 658-663. doi: 10.1016/j.foodchem.2015.11.013

Linares, D. M., Fernández, M., Martín, M. C., and Álvarez, M. A. (2009). Tyramine biosynthesis in Enterococcus durans is transcriptionally regulated by the extracellular $\mathrm{pH}$ and tyrosine concentration. Microb. Biotechnol. 2, 625-633. doi: 10.1111/j.1751-7915.2009.00117.x

Linares, D. M., Perez, M., Ladero, V., del Rio, B., Redruello, B., Martin, M., et al. (2014). An agmatine-inducible system for the expression of recombinant proteins in Enterococcus faecalis. Microb. Cell Fact. 13:169. doi: 10.1186/s12934014-0169-1

Livak, K. J., and Schmittgen, T. D. (2001). Analysis of relative gene expression data using real-time quantitative PCR and the $2^{-\Delta \Delta \mathrm{C}_{\mathrm{T}}}$ method. Methods 25 , 402-408. doi: 10.1006/meth.2001.1262

Mader, U., Homuth, G., Scharf, C., Buttner, K., Bode, R., and Hecker, M. (2002). Transcriptome and proteome analysis of Bacillus subtilis gene expression modulated by amino acid availability. J. Bacteriol. 184, 4288-4295. doi: 10.1128/ JB.184.15.4288-4295.2002

Mazzoli, R., Pessione, E., Dufour, M., Laroute, V., Giuffrida, M. G., Giunta, C., et al. (2010). Glutamate-induced metabolic changes in Lactococcus lactis NCDO 2118 during GABA production: combined transcriptomic and proteomic analysis. Amino Acids 39, 727-737. doi: 10.1007/s00726-010-0507-5

Paulsen, I. T., Banerjei, L., Myers, G. S., Nelson, K. E., Seshadri, R., Read, T. D., et al. (2003). Role of mobile DNA in the evolution of vancomycin-resistant Enterococcus faecalis. Science 299, 2071-2074. doi: 10.1126/science.1080613

Perez, M., Calles-Enriquez, M., Nes, I., Martin, M. C., Fernandez, M., Ladero, V., et al. (2015). Tyramine biosynthesis is transcriptionally induced at low $\mathrm{pH}$ and improves the fitness of Enterococcus faecalis in acidic environments. Appl. Microbiol. Biotech. 99, 3547-3558. doi: 10.1007/s00253-014-6301-7

Perez, M., Ladero, V., Del Rio, B., Redruello, B., De Jong, A., Kuipers, O. P., et al. (2016). Transcriptome profiling of TDC cluster deletion mutant of Enterococcus faecalis V583. Genom Data 9, 67-69. doi: 10.1016/j.gdata.2015.10.002

Pessione, E. (2012). Lactic acid bacteria contribution to gut microbiota complexity: lights and shadows. Front. Cell Infect. Microbiol. 2:86. doi: 10.3389/fcimb.2012. 00086

Pessione, E., Pessione, A., Lamberti, C., Coisson, D. J., Riedel, K., Mazzoli, R., et al. (2009). First evidence of a membrane-bound, tyramine and betaphenylethylamine producing, tyrosine decarboxylase in Enterococcus faecalis: a two-dimensional electrophoresis proteomic study. Proteomics 9, 2695-2710. doi: $10.1002 /$ pmic. 200800780

Redruello, B., Ladero, V., Cuesta, I., Alvarez-Buylla, J. R., Martin, M. C., Fernandez, M., et al. (2013). A fast, reliable, ultra high performance liquid chromatography method for the simultaneous determination of amino acids, biogenic amines and ammonium ions in cheese, using diethyl ethoxymethylenemalonate as a derivatising agent. Food Chem. 139, 1029-1035. doi: $10.1016 /$ j.foodchem.2013.01.071

Russo, P., Fernández, de Palencia P, Romano, A., Fernández, M., Lucas, P., Spano, G., et al. (2012). Biogenic amine production by the wine Lactobacillus brevis IOEB 9809 in systems that partially mimic the gastrointestinal tract stress. BMC Microbiol. 12:247. doi: 10.1186/1471-2180-12-247

Sahm, D. F., Kissinger, J., Gilmore, M. S., Murray, P. R., Mulder, R., Solliday, J., et al. (1989). In vitro susceptibility studies of vancomycin-resistant Enterococcus faecalis. Antimicrob. Agents Chemother. 33, 1588-1591. doi: 10.1128/AAC.33.9. 1588

Suarez, C., Espariz, M., Blancato, V. S., and Magni, C. (2013). Expression of the agmatine deiminase pathway in Enterococcus faecalis is activated by the AguR regulator and repressed by CcpA and PTS ${ }^{\mathrm{Man}}$ systems. PLOS ONE 8:e76170. doi: 10.1371/journal.pone.0076170

Teixeira, J. S., Seeras, A., Sanchez-Maldonado, A. F., Zhang, C., Su, M. S., and Ganzle, M. G. (2014). Glutamine, glutamate, and arginine-based acid resistance in Lactobacillus reuteri. Food Microbiol. 42, 172-180. doi: 10.1016/j.fm.2014. 03.015

ten Brink, B., Damink, C., Joosten, H. M., and Huis in 'T Veld, J. H. J. (1990). Occurrence and formation of biologically active amines in foods. Int. J. Food Microbiol. 11, 73-84. doi: 10.1016/0168-1605(90)90040-C

Conflict of Interest Statement: The authors declare that the research was conducted in the absence of any commercial or financial relationships that could be construed as a potential conflict of interest.

Copyright (๐ 2017 Perez, Ladero, del Rio, Redruello, de Jong, Kuipers, Kok, Martin, Fernandez and Alvarez. This is an open-access article distributed under the terms of the Creative Commons Attribution License (CC BY). The use, distribution or reproduction in other forums is permitted, provided the original author(s) or licensor are credited and that the original publication in this journal is cited, in accordance with accepted academic practice. No use, distribution or reproduction is permitted which does not comply with these terms. 